\title{
Weak lensing of the cosmic microwave background by foreground gravitational waves
}

\author{
Chao $\mathrm{Li}^{1}$ and Asantha Cooray ${ }^{2}$ \\ ${ }^{1}$ Theoretical Astrophysics, California Institute of Technology, Mail Code 103-33, Pasadena, California 91125, USA \\ ${ }^{2}$ Center for Cosmology, Department of Physics and Astronomy, University of California, 4129 Frederick Reines Hall, \\ Irvine, California 92697, USA
}

(Received 14 April 2006; published 19 July 2006)

\begin{abstract}
Weak lensing distortion of the background cosmic microwave background (CMB) temperature and polarization patterns by the foreground density fluctuations is well studied in the literature. We discuss the gravitational lensing modification to $\mathrm{CMB}$ anisotropies and polarization by a stochastic background of primordial gravitational waves between us and the last scattering surface. While density fluctuations perturb CMB photons via gradient-type deflections only, foreground gravitational waves distort CMB anisotropies via both gradient- and curl-type displacements. The latter is a rotation of background images, while the former is related to the lensing convergence. For a primordial background of inflationary gravitational waves, with an amplitude corresponding to a tensor-to-scalar ratio below the current upper limit of $\sim 0.3$, the resulting modifications to the angular power spectra of CMB temperature anisotropy and polarization are below the cosmic variance limit. At tens of arcminute angular scales and below, these corrections, however, are above the level at which systematics must be controlled in all-sky anisotropy and polarization maps with no instrumental noise and other secondary and foreground signals.
\end{abstract}

PACS numbers: 98.80.Es, 95.85.Nv, 98.35.Ce, 98.70.Vc

\section{INTRODUCTION}

The weak lensing of cosmic microwave background (CMB) anisotropies and polarization by intervening mass fluctuations, or scalar perturbations, is now well studied in the literature [1,2], with a significant effort spent on improving the accuracy of analytical and numerical calculations (see, recent review in [3]). The non-Gaussian pattern of CMB anisotropies and polarization created by nonlinear mapping associated with lensing angular deflections aids the extraction of certain statistical properties of the foreground mass distribution [4]. Weak lensing deflections by intervening mass also leak $\mathrm{CMB}$ polarization power in the $E$-mode to the $B$-mode [5]. This lensing $B$-mode signal presents a significant confusion when searching for primordial gravitational wave signatures in the $\mathrm{CMB}$ polarization [6]. The lensing reconstruction techniques discussed in the literature, however, allow the possibility to "clean" CMB polarization maps and to search for a background of inflationary gravitational waves with an energy scale as low as $10^{15} \mathrm{GeV}$ [7].

Similar to gravitational lensing by density perturbations, if there is a background of gravitational waves in the foreground, then one would expect metric perturbations associated with these waves to distort and gravitationally lens background images [8]. While the lensing deflections by the density field can be written as the gradient of the projected gravitational potential, lensing displacements due to gravitational waves can be decomposed to both a gradient and a curl-like component [9-11]. In these two components, gradient-type displacements are related to the lensing convergence, while curl-type displacements are related to the image rotation, though both types of displacements lead to image shear. While linear density per- turbations do not produce rotations, second-order corrections to weak lensing by scalar perturbations, such as due to the coupling of two lenses along the line of sight, can produce rotational modes [12].

While the study of CMB lensing by foreground density fluctuations is now well developed [3], the discussion of CMB lensing by foreground gravitational waves is limited. In the context of large-scale structure weak lensing surveys with galaxy shapes [13], the rotational power spectrum of background galaxy images when lensed by primordial gravitational waves in the foreground is discussed in Ref. [10]. In the context of lensing reconstruction with CMB temperature and polarization maps, the curl component of the displacement field can be used to monitor systematics [11], though lensing by gravitational waves will leave a nonzero contribution to the curl component.

Here, we extend the calculation in Ref. [10] and study both the curl and the gradient modes of the deflection field from primordial gravitational waves that intervene CMB photons propagating from the last scattering surface. Our calculations are both useful and important given the increasing interest on, and plans for, high sensitivity CMB anisotropy and polarization measurements, including a potential space-based mission after Planck, called CMBpol in the future. Such an experiment is expected to study polarization $B$-modes in exquisite detail and it is important to understand potentially interesting secondary signals beyond those that are routinely mentioned in the literature. Based on the calculations presented here, unfortunately, we find that gravitational lensing of CMB by a background of primordial gravitational waves from inflation, with an amplitude below the current tensor-to-scalar ratio upper limit of 0.3 , will produce an undetectable modification to anisotropy and polarization power spectra. 
Moreover, since the corrections are below the cosmic variance level, it is unlikely that one needs to account for these secondary corrections when making precise cosmological measurements.

This paper is organized as follows. In Sec. II, we discuss lensing by foreground gravitational waves by discussing both the gradient and curl components of the displacement field. Section III presents expressions for the weak lensing correction to the CMB anisotropy and polarization power spectra. We conclude with a discussion of our results in Sec. IV.

\section{THE SPECTRUM OF EXPANSION AND ROTATION}

To establish the lensing correction to CMB anisotropy and polarization maps by foreground gravitational waves, we first need to calculate the photon displacement on the spherical sky by gravitational waves in the foreground. We make use of synchronous coordinates and take the metric of a Friedman-Robertson-Walker cosmological model as

$$
g_{\mu \nu}=a^{2}\left(\begin{array}{cc}
-1 & 0 \\
0 & \mathbf{I}+\mathbf{H}
\end{array}\right),
$$

where the scale factor is $a(\eta)$, and the conformal time is denoted by $\eta$ with the value today of $\eta_{0}$. Here, $\mathbf{H}$ is the transverse $(\nabla \cdot \mathbf{H}=0)$, symmetric $\left(\mathbf{H}=\mathbf{H}^{\mathrm{T}}\right)$, and traceless $(\operatorname{Tr} \mathbf{H}=0)$ tensor metric perturbation associated with gravitational waves, while $\mathbf{I}$ is the identity matrix. The photon propagation is governed by the geodesic equation

$$
\frac{d^{2} x^{\mu}}{d \lambda^{2}}+\Gamma_{\alpha \beta}^{\mu} \frac{d x^{\alpha}}{d \lambda} \frac{d x^{\beta}}{d \lambda}=0 .
$$

By changing variables to the conformal time $\eta$ from the affine parameter $\lambda$, the geodesic equation in the presence of foreground gravitational waves is [10]

$$
\begin{aligned}
\ddot{\mathbf{r}}= & \frac{1}{2}(\dot{\mathbf{r}} \cdot \dot{\mathbf{H}} \cdot \dot{\mathbf{r}}) \dot{\mathbf{r}}-(\mathbf{I}+\mathbf{H})^{-1} \\
& \cdot\left[\dot{\mathbf{r}} \cdot \frac{d}{d \eta} \mathbf{H}-\frac{1}{2} \nabla_{H}(\dot{\mathbf{r}} \cdot \mathbf{H} \cdot \dot{\mathbf{r}})\right],
\end{aligned}
$$

where, to simplify the notation, we have not written out the explicit dependence of $\eta$ in each of these terms. Here, the overdot represents the derivative with respect to the conformal time. The full derivative $d / d \eta$ can be separated to $\partial / \partial \eta+\dot{\mathbf{r}} \cdot \nabla$. Here, and throughout, $\nabla_{H}$ denotes the gradient that applies only to the metric perturbation $\mathbf{H}$; when not subscripted with $H$, the gradient should be interpreted as the one that applies to all terms, including the line-ofsight directional vector $\mathbf{n}$.

As gravitational fluctuation is very weak today, hereafter we neglect $\mathbf{H}\left(\eta_{0}\right)$, and choose the initial conditions of the trajectory to be

$$
\mathbf{r}\left(\eta_{0}\right)=0, \quad \dot{\mathbf{r}}\left(\eta_{0}\right)=-\hat{\mathbf{n}} .
$$

We find that the general displacement on the celestial sphere induced by primordial gravitational waves is

$$
\begin{aligned}
\mathbf{r}(\eta)= & \hat{\mathbf{n}}\left(\eta_{0}-\eta\right)-\int_{\eta}^{\eta_{0}} d \eta^{\prime}\left(\mathbf{H} \cdot \hat{\mathbf{n}}+\frac{1}{2}\left(\eta^{\prime}-\eta\right)\right. \\
& \left.\times\left[(\hat{\mathbf{n}} \cdot \dot{\mathbf{H}} \cdot \hat{\mathbf{n}}) \hat{\mathbf{n}}-\nabla_{H}(\hat{\mathbf{n}} \cdot \mathbf{H} \cdot \hat{\mathbf{n}})\right]\right)_{\left(\eta^{\prime}, \mathbf{x}^{\prime}\right)}
\end{aligned}
$$

where $\mathbf{x}^{\prime}=\left(\eta_{0}-\eta^{\prime}\right) \hat{\mathbf{n}}$. Similar to Ref. [10], we have evaluated the trajectory on the unperturbed path following the so-called Born approximation. One could potentially evaluate corrections to this approximation in terms of a perturbative correction to the path length, but these would be at the second order in metric perturbations and will be ignored.

The total displacement can be separated to a part along the line of sight and a part perpendicular to it. The radial displacement leads to a time-delay effect, similar to the lensing time delay associated with foreground potentials [14]. This time delay couples to the radial gradient of the $\mathrm{CMB}$, due to finite extent of the last scattering surface, while the angular displacement couples to the angular gradient. As discussed in Ref. [15], the overall correction to CMB anisotropy spectra from the time-delay effect is subdominant since the spatial gradient of the CMB is, at least, 2 orders of magnitude smaller compared to the angular gradient. There are also geometrical cancellations that make the time-delay effect smaller relative to angular deflections. Thus, we ignore the radial displacement and only consider the transverse component related to gravitational lensing angular deflections.

Using the transverse displacement, the angular deflection projected on the spherical sky is $\vec{\Delta}=[\mathbf{r}(\eta)-(\hat{\mathbf{n}}$. $\mathbf{r}) \hat{\mathbf{n}}] /\left(\eta_{0}-\eta\right)$. These two-dimensional displacements can be related to usual quantities in gravitational lensing with the convergence and the rotation defined as [9]

$$
\kappa \equiv-\frac{\Delta^{a}: a}{2}, \quad \text { and } \quad \omega \equiv \frac{\left(\Delta_{a} \epsilon^{a b}\right)_{: b}}{2},
$$

respectively.

Similarly, we note that the general displacement on the celestial sphere can be decomposed to two components,

$$
\Delta_{a}=-\sum_{l m}\left(h_{l m}^{\oplus} Y_{l m: a}+h_{l m}^{\otimes} Y_{l m: b} \epsilon_{a}^{b}\right),
$$

where

$$
\begin{aligned}
& h_{l m}^{\oplus}=\frac{1}{l(l+1)} \int d \hat{\mathbf{n}} Y_{l m}^{*} \Delta_{a}: a=-\frac{2}{l(l+1)} \int d \hat{\mathbf{n}} Y_{l m}^{*} \kappa \\
& h_{l m}^{\otimes}=\frac{1}{l(l+1)} \int d \hat{\mathbf{n}} Y_{l m}^{*} \Delta_{a}: b \boldsymbol{\epsilon}_{b}^{a}=\frac{2}{l(l+1)} \int d \hat{\mathbf{n}} Y_{l m}^{*} \omega,
\end{aligned}
$$

where $\oplus$ and $\otimes$ denote the gradient- and curl-type deflections, respectively. For simplicity, we have dropped the dependence on the directional vector parametrized by $\hat{\mathbf{n}}$. 
The lensing by foreground density perturbations to the first order only leads to a gradientlike displacement, while both components are generated when lensed by gravitational waves. We now calculate both the convergence and the rotational spectrum of the displacement field due to foreground stochastic gravitational waves.

\section{A. Gradient spectrum}

Gradient deflections are associated with the expansion and, as defined above, can be described in terms of the convergence: $\kappa(\hat{\mathbf{n}}) \equiv-\Delta^{a}: a / 2$. In general the transverse divergence of a vector $\vec{A}$ can be rewritten as

$$
\begin{aligned}
& \frac{1}{\sin \theta} \frac{\partial}{\partial \theta}\left(\sin \theta A_{\theta}\right)+\frac{1}{\sin \theta} \frac{\partial A_{\phi}}{\partial \phi} \\
& \quad=r\left(\nabla \cdot \vec{A}-(\hat{\mathbf{n}} \cdot \nabla)(\hat{\mathbf{n}} \cdot \vec{A})-\frac{2 \hat{\mathbf{n}} \cdot \vec{A}}{r}\right),
\end{aligned}
$$

where $r=\eta_{0}-\eta^{\prime}$. When substituting the form of $\mathbf{r}(\eta)$ from Eq. (5), the gradient terms here lead to terms that are due to $\nabla \cdot \hat{\mathbf{n}}$ and $\nabla \cdot \mathbf{H}$. We first consider the former and, making use of the fact that $\partial_{i} \hat{n}_{j}=\left(\delta_{i j}-\hat{n}_{i} \hat{n}_{j}\right) /\left(\eta_{0}-\eta^{\prime}\right)$, we separate contributions to convergence to two components and write $\kappa(\hat{\mathbf{n}})=\kappa_{1}+\kappa_{2}$ as

$$
\begin{aligned}
\kappa_{1}= & -\frac{1}{2} \int_{\eta_{s}}^{\eta_{0}} d \eta^{\prime}\left(\frac{\eta_{0}-\eta^{\prime}}{\eta_{0}-\eta_{s}}\right)\left(\hat{\mathbf{n}} \cdot \nabla_{H}\right)(\hat{\mathbf{n}} \cdot \mathbf{H} \cdot \hat{\mathbf{n}})-\frac{3}{2\left(\eta_{0}-\eta_{s}\right)} \int_{\eta_{s}}^{\eta_{0}} d \eta^{\prime}(\hat{\mathbf{n}} \cdot \mathbf{H} \cdot \hat{\mathbf{n}}), \\
\kappa_{2}= & -\frac{1}{4} \int_{\eta_{s}}^{\eta_{0}} d \eta^{\prime}\left(\frac{\eta_{0}-\eta^{\prime}}{\eta_{0}-\eta_{s}}\right)\left(\eta^{\prime}-\eta_{s}\right) \nabla_{H}^{2}(\hat{\mathbf{n}} \cdot \mathbf{H} \cdot \hat{\mathbf{n}})+\frac{1}{4} \int_{\eta_{s}}^{\eta_{0}} d \eta^{\prime}\left(\frac{\eta_{0}-\eta^{\prime}}{\eta_{0}-\eta_{s}}\right)\left(\eta^{\prime}-\eta_{s}\right)\left(\hat{\mathbf{n}} \cdot \nabla_{H}\right)^{2}(\hat{\mathbf{n}} \cdot \mathbf{H} \cdot \hat{\mathbf{n}}) \\
& +\frac{1}{\eta_{0}-\eta_{s}} \int_{\eta_{s}}^{\eta_{0}} d \eta^{\prime}\left(\eta^{\prime}-\eta_{s}\right)\left(\hat{\mathbf{n}} \cdot \nabla_{H}\right)(\hat{\mathbf{n}} \cdot \mathbf{H} \cdot \hat{\mathbf{n}}),
\end{aligned}
$$

where we have explicitly simplified the calculation by including terms associated with $\nabla \cdot \hat{\mathbf{n}}$.

Note that we have also replaced $\eta \rightarrow \eta_{s}$ corresponding to the conformal time at the last scattering surface of CMB. To simplify, we decompose the metric perturbation into the Fourier component,

$$
\mathbf{H}(\mathbf{x}, \eta)=\frac{1}{(2 \pi)^{3 / 2}} \int d^{3} \mathbf{k} e^{i \mathbf{k} \cdot \mathbf{x}} T(k, \eta) \sum_{j=1}^{2} H_{j}(\mathbf{k}) \mathbf{e}_{j}(\mathbf{k}),
$$

where we have introduced the gravitational wave transfer function that describes the time evolution of the metric perturbation with $\mathbf{H}_{j}(\mathbf{k}, \eta)=T(k, \eta) H_{j}(\mathbf{k}) \mathbf{e}_{j}(\mathbf{k})$.

The terms in Eq. (10) can be simplified as

$$
\begin{aligned}
\kappa_{1}= & -\frac{1}{2} \int_{\eta_{s}}^{\eta_{0}} d \eta^{\prime} \frac{1}{(2 \pi)^{3 / 2}} \int d^{3} \mathbf{k} e^{i \mathbf{k} \cdot \mathbf{x}^{\prime}} T\left(k, \eta^{\prime}\right)\left(\frac{\eta_{0}-\eta^{\prime}}{\eta_{0}-\eta_{s}}\right) \times \sum_{j} H_{j}(\mathbf{k})\left[\hat{\mathbf{n}} \cdot \mathbf{e}_{j}(\mathbf{k}) \cdot \hat{\mathbf{n}}\right](i \mathbf{k} \cdot \hat{\mathbf{n}}) \\
& -\frac{3}{2\left(\eta_{0}-\eta_{s}\right)} \int_{\eta_{s}}^{\eta_{0}} d \eta^{\prime} \frac{1}{(2 \pi)^{3 / 2}} \int d^{3} \mathbf{k} e^{i \mathbf{k} \cdot \mathbf{x}^{\prime}} T\left(k, \eta^{\prime}\right) \sum_{j} H_{j}(\mathbf{k})\left[\hat{\mathbf{n}} \cdot \mathbf{e}_{j}(\mathbf{k}) \cdot \hat{\mathbf{n}}\right] \\
\kappa_{2}= & \int_{\eta_{s}}^{\eta_{0}} d \eta^{\prime} \frac{\eta^{\prime}-\eta_{s}}{4} \frac{1}{(2 \pi)^{3 / 2}} \int d^{3} \mathbf{k} e^{i \mathbf{k} \cdot \mathbf{x}^{\prime}} T\left(k, \eta^{\prime}\right)\left(\frac{\eta_{0}-\eta^{\prime}}{\eta_{0}-\eta_{s}}\right) \times \sum_{j} \mathbf{H}_{j}(\mathbf{k})\left[\mathbf{k}^{2}-(\hat{\mathbf{n}} \cdot \mathbf{k})^{2}\right]\left[\hat{\mathbf{n}} \cdot \mathbf{e}_{j}(\mathbf{k}) \cdot \hat{\mathbf{n}}\right] \\
& +\frac{1}{\eta_{0}-\eta_{s}} \int_{\eta_{s}}^{\eta_{0}} d \eta^{\prime}\left(\eta^{\prime}-\eta_{s}\right) \frac{1}{(2 \pi)^{3 / 2}} \int d^{3} \mathbf{k} e^{i \mathbf{k} \cdot \mathbf{x}^{\prime}} T\left(k, \eta^{\prime}\right) \sum_{j} H_{j}(\mathbf{k})\left[\hat{\mathbf{n}} \cdot \mathbf{e}_{j}(\mathbf{k}) \cdot \hat{\mathbf{n}}\right](i \mathbf{k} \cdot \hat{\mathbf{n}}) .
\end{aligned}
$$

Here, $\mathbf{x}^{\prime}=\left(\eta_{0}-\eta^{\prime}\right) \hat{\mathbf{n}}$ and $\mathbf{e}_{j}$ represents the symmetric, traceless polarization tensor that obeys $\operatorname{Tr}\left[\mathbf{e}_{j}(\mathbf{k}) \cdot \mathbf{e}_{k}(\mathbf{k})\right]=$ $2 \delta_{j k}$ and $\mathbf{k} \cdot \mathbf{e}_{j}(\mathbf{k})=0$ with $j$ in Eq. (12) summing over the two linear polarization states.

Since gravitational waves trace the wave equation with

$$
\ddot{\mathbf{H}}-\nabla^{2} \mathbf{H}+2 \frac{\dot{a}}{a} \mathbf{H}=16 \pi G a^{2} \mathbf{P},
$$

where $\mathbf{P}$ is the tensor part of the anisotropic stress, say from neutrinos (see Ref. [16] for details). The term on the right- hand side acts as a damping term for the evolution of gravitational waves and is important for modes that enter the horizon before matter-radiation equality, with a smaller correction for modes that enter the horizon after matterradiation equality. Since these corrections are not more than $30 \%$, while the amplitude of the gravitational wave background is uncertain to more than orders of magnitude, we ignore such subtleties here assuming no anisotropic stress; for a cosmological model dominated by matter, in Fourier space, one can write the evolution of $\mathbf{H}$ in terms of 
the transfer function as $T(k, \eta)=3 j_{1}(k \eta) /(k \eta)$. Though this transfer function is valid for large scales outside the horizon at matter-radiation equality, the inclusion of a more appropriate transfer function [16] did not change our results considerably as the overall lensing is dominated by long wavelength modes.

We define the power spectrum of metric perturbations as

$$
\left\langle H_{i}(\mathbf{k}) H_{j}^{*}(\mathbf{k})\right\rangle=(2 \pi)^{3} P_{T}(k) \delta_{i j} \delta^{(3)}\left(\mathbf{k}-\mathbf{k}^{\prime}\right),
$$

where we assume isotropy and equal density of gravitational waves in the two polarization states $i$ and $j$. Following Ref. [10], we normalize the power spectrum to the Hubble parameter during inflation and take

$$
P_{T}(k)=\frac{8 \pi}{(2 \pi)^{3}}\left(\frac{H_{I}}{M_{\text {Planck }}}\right)^{2} k^{-3} .
$$

Using this three-dimensional power spectrum for metric perturbations, the angular power spectrum of gradient-type deflections is

$$
\begin{aligned}
C_{l}^{h^{\oplus}} & =\frac{1}{2 l+1} \sum_{m=-l}^{m=l}\left\langle\left|h_{l m}^{\oplus}\right|^{2}\right\rangle \\
& =\frac{4}{(2 l+1) l^{2}(l+1)^{2}} \sum_{m=-l}^{m=l}\left\langle\left|\int d \hat{\mathbf{n}} Y_{l m}^{*}(\hat{\mathbf{n}}) \kappa(\hat{\mathbf{n}})\right|^{2}\right\rangle \\
& =\frac{\pi}{l^{2}(l+1)^{2}} \frac{(l+2) !}{(l-2) !} \int d^{3} \mathbf{k} P_{T}(k)\left|T_{1}+T_{2}+T_{m}^{\oplus}\right|^{2},
\end{aligned}
$$

where the terms are again

$$
\begin{aligned}
T_{1}= & -k \int_{\eta_{s}}^{\eta_{0}} d \eta^{\prime}\left(\frac{\eta_{0}-\eta^{\prime}}{\eta_{0}-\eta_{s}}\right) T\left(k, \eta^{\prime}\right)\left[\left.\partial_{x}\left(x^{-2} j_{l}(x)\right)\right|_{x=k\left(\eta_{0}-\eta^{\prime}\right)}\right]-\left.\frac{3}{\eta_{0}-\eta_{s}} \int_{\eta_{s}}^{\eta_{0}} d \eta^{\prime} T\left(k, \eta^{\prime}\right)\left(x^{-2} j_{l}(x)\right)\right|_{x=k\left(\eta_{0}-\eta^{\prime}\right),}, \\
T_{2}= & \frac{k^{2}}{2} \int_{\eta_{s}}^{\eta_{0}} d \eta^{\prime}\left(\frac{\eta_{0}-\eta^{\prime}}{\eta_{0}-\eta_{s}}\right)\left(\eta^{\prime}-\eta_{s}\right) T\left(k, \eta^{\prime}\right)\left[\left.\left(1+\partial_{x}^{2}\right)\left(x^{-2} j_{l}(x)\right)\right|_{x=k\left(\eta_{0}-\eta^{\prime}\right)}\right] \\
& +\frac{2 k}{\eta_{0}-\eta_{s}} \int_{\eta_{s}}^{\eta_{0}} d \eta^{\prime}\left(\eta^{\prime}-\eta_{s}\right) T\left(k, \eta^{\prime}\right)\left[\left.\partial_{x}\left(x^{-2} j_{l}(x)\right)\right|_{x=k\left(\eta_{0}-\eta^{\prime}\right)}\right] .
\end{aligned}
$$

Note that the convergence power spectrum due to gravitational wave deflections is $C_{l}^{\kappa \kappa}=l^{2}(l+1)^{2} C_{l}^{h^{\oplus}} / 4$.

In Eq. (16), we have also introduced an additional correction to the gradient-type deflection spectrum with the term $T_{m}^{\oplus}$. As discussed in Ref. [10], under the assumption that the background source is isotropic, this term corrects for the metric shear at the source plane. In our calculation, this is equivalent to assuming that the hot and cold spots in the CMB map are not sheared by the gravitational waves present at the last scattering surface. If the background source is sheared, then this correction is unnecessary. Since we have not carried out a detailed secondorder or higher perturbation-theory calculation on the effects of gravitational waves at the last scattering surface to check if the primordial features on the CMB map are sheared or not, as in Ref. [10], we will include this term as an overall correction to the lensing calculation. To test our assumption, we encourage a detailed perturbationtheory calculation on whether this term is necessary or not.

Note that the term in Eq. (16) is associated with the gradient-type deflection pattern or convergence of the correction term. Later, we will also correct the curl-type deflections or rotations as well. To calculate this correction, we first evaluate the displacement vector at the last scattering surface

$$
\mathbf{r}_{m}=-\frac{\mathbf{H}}{2} \cdot \hat{\mathbf{n}}\left(\eta_{0}-\eta_{s}\right)
$$

and then project this displacement vector to obtain the transverse displacement vector of

$$
\Delta_{m}=\frac{\mathbf{r}_{m}-\left(\mathbf{r}_{m} \cdot \hat{\mathbf{n}}\right) \hat{\mathbf{n}}}{\eta_{0}-\eta_{s}} .
$$

This vector can be decomposed to the gradient- and curltypes deflections. Making use of the fact that the convergence is $\kappa(\hat{\mathbf{n}}) \equiv-\Delta_{m: a}^{a} / 2$ and taking the Fourier transforms, we write the required term in Eq. (16) as

$$
\begin{aligned}
T_{m}^{\oplus}= & -\frac{k}{2}\left(\eta_{0}-\eta_{s}\right) T\left(k, \eta_{s}\right)\left[\left.\partial_{x}\left(x^{-2} j_{l}(x)\right)\right|_{x=k\left(\eta_{0}-\eta_{s}\right)}\right] \\
& -\left.T\left(k, \eta_{s}\right)\left(x^{-2} j_{l}(x)\right)\right|_{x=k\left(\eta_{0}-\eta_{s}\right) .}
\end{aligned}
$$

This completes the calculation of gradient-type deflection power spectrum by taking into account the lensing by intervening gravitational waves between the last scattering surface and the observer and the metric-shear correction related to gravitational waves present at the last scattering surface from which photons propagate.

\section{B. Curl spectrum}

Rotation is defined to be $\omega(\hat{\mathbf{n}}) \equiv \frac{1}{2}\left(\Delta_{a} \epsilon^{a b}\right)_{: b}$, which leads to

$$
\omega(\hat{\mathbf{n}}) \equiv-\frac{1}{2} \hat{\mathbf{n}} \cdot\left(\nabla \times \mathbf{r}\left(\hat{\mathbf{n}}, \eta_{s}\right)\right) .
$$

Equation (6) gives

$$
\omega=\frac{1}{2} \int_{\eta_{s}}^{\eta_{0}} d \eta^{\prime}[\hat{\mathbf{n}} \cdot(\nabla \times \mathbf{H}) \cdot \hat{\mathbf{n}}],
$$

since $\nabla \times \hat{\mathbf{n}}=0$. Here we define the curl of the second rank tensor $\mathbf{H}$ by $(\nabla \times \mathbf{H})_{i l}=\epsilon_{i j k} \partial_{j} H_{k l}$. 
The curl-type deflection spectrum from this term is [10]

$$
\begin{aligned}
C_{l}^{h^{\otimes}} & =\frac{1}{2 l+1} \sum_{m=-l}^{m=l}\left\langle\left|h_{l m}^{\otimes}\right|^{2}\right\rangle \\
& =\frac{4}{(2 l+1) l^{2}(l+1)^{2}} \sum_{m=-l}^{m=l}\left\langle\left|\int d \hat{\mathbf{n}} Y_{l m}^{*}(\hat{\mathbf{n}}) \omega(\hat{\mathbf{n}})\right|^{2}\right\rangle \\
& =\frac{\pi}{l^{2}(l+1)^{2}} \frac{(l+2) !}{(l-2) !} \int d^{3} \mathbf{k} P_{T}(k)\left|T_{3}+T_{m}^{\otimes}\right|^{2}
\end{aligned}
$$

where

$$
T_{3}=\left.2 k \int_{\eta_{s}}^{\eta_{0}} d \eta^{\prime} T\left(k, \eta^{\prime}\right)\left(x^{-2} j_{l}(x)\right)\right|_{x=k\left(\eta_{0}-\eta^{\prime}\right)} .
$$

Again, one can write the power spectrum of rotation as $C_{l}^{\omega \omega}=l^{2}(l+1)^{2} C_{l}^{h^{\otimes}} / 4$.

Here also we include the correction to the rotational spectrum due to metric perturbations at the last scattering surface. Similar to convergence, by following the same procedure as before but taking $1 / 2\left(\left[\Delta_{m}\right]_{a} \epsilon^{a b}\right)_{: b}$ of Eq. (19), we get

$$
T_{m}^{\otimes}=\left.k\left(\eta_{0}-\eta_{s}\right) T\left(k, \eta_{s}\right)\left(x^{-2} j_{l}(x)\right)\right|_{x=k\left(\eta_{0}-\eta_{s}\right.} .
$$

For comparison, we note that density fluctuations along the line of sight lead to gradient-type deflections only. The resulting contributions are described in terms of the angular power spectrum of projected potential $C_{l}^{\phi \phi}$ that is well studied in the literature [2]. We do not repeat those derivations here, but will provide a comparison of lensing under gravitational waves and lensing by mass in the discussion later.

\section{LENSING OF CMB BY GRAVITATIONAL WAVES}

In this section, we will discuss the analytical calculation related to how foreground gravitational waves modify the background CMB temperature anisotropy and polarization patterns. Here, we will concentrate on the angular power spectra of CMB observables. The lensing of CMB by foreground density fluctuations is formulated in Ref. [2] and we follow the same procedure here. Since deflection field power spectra peak at large angular scales, we present an analytical formulation as appropriate for the spherical sky. The calculation we consider here, however, is perturbative and one expects important corrections beyond the first order in the deflection angle. For foreground gravitational waves, such issues can be ignored as the overall modification to the anisotropy and polarization spectra is small.

\section{A. Temperature anisotropies}

Following Ref. [2], the lensed temperature field $\tilde{\theta}$ can be expressed as

$$
\begin{aligned}
\tilde{\theta}(\hat{\mathbf{n}}) & =\theta(\hat{\mathbf{n}}+\boldsymbol{\Delta}) \\
& =\theta(\hat{\mathbf{n}})+\nabla^{a} \theta \cdot \Delta_{a}+\frac{1}{2} \nabla^{b} \nabla^{a} \theta \cdot \Delta_{a} \Delta_{b},
\end{aligned}
$$

where $\theta(\hat{\mathbf{n}})$ is the unlensed temperature fluctuation in the direction $\hat{\mathbf{n}}$. The temperature field can be expanded to multipole moments such that $\theta(\hat{\mathbf{n}})=\sum_{l m} \theta_{l m} Y_{l m}(\hat{\mathbf{n}})$. Taking the spherical harmonic moment of Eq. (26) and using Eq. (7), we find

$$
\begin{aligned}
\tilde{\theta}_{l m}= & \theta_{l m}+\int d \hat{\mathbf{n}} Y_{l m}^{*}\left[\nabla^{a} \theta \cdot \Delta_{a}+\frac{1}{2} \nabla^{b} \nabla^{a} \theta \cdot \Delta_{a} \Delta_{b}\right] \\
= & \theta_{l m}-\sum_{l_{1} m_{1} l_{2} m_{2}}\left(I_{l m l_{1} m_{1} l_{2} m_{2}}^{\oplus} \theta_{l_{1} m_{1}} h_{l_{2} m_{2}}^{\oplus}+I_{l m l_{1} m_{1} l_{2} m_{2}}^{\otimes} \theta_{l_{1} m_{1}} h_{l_{2} m_{2}}^{\otimes}\right) \\
& +\frac{1}{2} \sum_{l_{1} m_{1} l_{2} m_{2} l_{3} m_{3}}\left(J_{l m l_{1} m_{1} l_{2} m_{2} l_{3} m_{3}}^{\oplus} \theta_{l_{1} m_{1}} h_{l_{2} m_{2}}^{\oplus} h_{l_{3} m_{3}}^{\oplus *}+J_{l m l_{1} m_{1} l_{2} m_{2} l_{3} m_{3}}^{\otimes} \theta_{l_{1} m_{1}} h_{l_{2} m_{2}}^{\otimes} h_{l_{3} m_{3}}^{\otimes *}\right),
\end{aligned}
$$

where the integrals are

$$
\begin{aligned}
I_{l m l_{1} m_{1} l_{2} m_{2}}^{\oplus} & =\int d \hat{\mathbf{n}} Y_{l m}^{*} Y_{l_{1} m_{1}}^{a} Y_{l_{2} m_{2}: a}, \\
I_{l m l_{1} m_{1} l_{2} m_{2}}^{\otimes} & =\int d \hat{\mathbf{n}} Y_{l m}^{*} Y_{l_{1} m_{1}}^{a} Y_{l_{2} m_{2}: b} \epsilon_{a}^{b} \\
J_{l m l_{1} m_{1} l_{2} m_{2} l_{3} m_{3}}^{\oplus} & =\int d \hat{\mathbf{n}} Y_{l m}^{*} Y_{l_{1} m_{1}}^{a b} Y_{l_{2} m_{2}: a} Y_{l_{3} m_{3}: b}^{*}, \\
J_{l m l_{1} m_{1} l_{2} m_{2} l_{3} m_{3}}^{\otimes} & =\int d \hat{\mathbf{n}} Y_{l m}^{*} Y_{l_{1} m_{1}}^{a b} Y_{l_{2} m_{2}: c} Y_{l_{3} m_{3}: d}^{*} \epsilon_{a}^{c} \epsilon_{b}^{d} .
\end{aligned}
$$

The lensed temperature anisotropy power spectrum is

$$
\begin{aligned}
C_{l}^{\tilde{\theta}}= & C_{l}^{\theta}+\sum_{l_{1} l_{2}} C_{l_{1}}^{\theta}\left(C_{l_{2}}^{h^{\oplus}} S_{1}^{\oplus}+C_{l_{2}}^{h^{\otimes}} S_{1}^{\otimes}\right) \\
& +C_{l}^{\theta} \sum_{l_{1}}\left(C_{l_{1}}^{h^{\oplus}} S_{2}^{\oplus}+C_{l_{1}}^{h^{\otimes}} S_{2}^{\otimes}\right)
\end{aligned}
$$

where

$$
\begin{gathered}
S_{1}^{\oplus}=\sum_{m_{1} m_{2}}\left|I_{l m l_{1} m_{1} l_{2} m_{2}}\right|^{2} \quad S_{1}^{\otimes}=\sum_{m_{1} m_{2}}\left|I_{l m l_{1} m_{1} l_{2} m_{2}}^{\otimes}\right|^{2} \\
S_{2}^{\oplus}=\frac{1}{2} \sum_{m_{1}} J_{l m l m l_{1} m_{1} l_{1} m_{1}}^{\oplus}+\text { c.c. } \\
S_{2}^{\otimes}=\frac{1}{2} \sum_{m_{1}} J_{l m l m l_{1} m_{1} l_{1} m_{1}}^{\otimes}+\text { c.c., }
\end{gathered}
$$

and c.c. is the complex conjugate. The terms $S_{1}^{\oplus}$ and $S_{2}^{\oplus}$ are 
similar to those involving lensing by foreground density perturbations [2]. First, the integral $I_{l m l_{1} m_{1} l_{2} m_{2}}$ can be simplified through integration by parts and noting $\nabla^{2} Y_{l m}=-l(l+1) Y_{l m}$ and the general integral of three spin-spherical harmonics over the sky:

$$
\int d \hat{\mathbf{n}}\left({ }_{s_{1}} Y_{l_{1} m_{1}}^{*}\right)\left({ }_{s_{2}} Y_{l_{2} m_{2}}\right)\left({ }_{s_{3}} Y_{l_{3} m_{3}}\right)=(-1)^{m_{1}+s_{1}} \sqrt{\frac{\left(2 l_{1}+1\right)\left(2 l_{2}+1\right)\left(2 l_{3}+1\right)}{4 \pi}}\left(\begin{array}{ccc}
l_{1} & l_{2} & l_{3} \\
s_{1} & -s_{2} & -s_{3}
\end{array}\right)\left(\begin{array}{ccc}
l_{1} & l_{2} & l_{3} \\
-m_{1} & m_{2} & m_{3}
\end{array}\right)
$$

when $s_{1}=s_{2}+s_{3}$. We note that under parity inversion, ${ }_{s} Y_{l m} \rightarrow(-1)_{-s}^{l} Y_{l m}$, which is a useful property when we discuss lensing modifications to the CMB polarization field.

With $s_{i}=0$ and noting that ${ }_{0} Y_{l m}=Y_{l m}$,

$$
I_{l m l_{1} m_{1} l_{2} m_{2}}^{\oplus}=\frac{1}{2}\left[l_{1}\left(l_{1}+1\right)+l_{2}\left(l_{2}+1\right)-l(l+1)\right](-1)^{m} \sqrt{\frac{(2 l+1)\left(2 l_{1}+1\right)\left(2 l_{2}+1\right)}{4 \pi}}\left(\begin{array}{ccc}
l & l_{1} & l_{2} \\
0 & 0 & 0
\end{array}\right)\left(\begin{array}{ccc}
l & l_{1} & l_{2} \\
-m & m_{1} & m_{2}
\end{array}\right) .
$$

Using the orthonormality relation of Wigner- $3 \mathrm{j}$ symbols

$$
\sum_{m_{1} m_{2}}\left(\begin{array}{ccc}
l_{1} & l_{2} & l_{3} \\
m_{1} & m_{2} & m_{3}
\end{array}\right)\left(\begin{array}{ccc}
l_{1} & l_{2} & l_{3} \\
m_{1} & m_{2} & m_{3}
\end{array}\right)=\frac{1}{2 l_{3}+1},
$$

we can write

$$
S_{1}^{\oplus}=\frac{1}{2 l+1}\left(F_{l l_{1} l_{2}}^{\oplus}\right)^{2}
$$

with

$$
F_{l l_{1} l_{2}}^{\oplus}=\frac{1}{2}\left[l_{1}\left(l_{1}+1\right)+l_{2}\left(l_{2}+1\right)-l(l+1)\right] \sqrt{\frac{(2 l+1)\left(2 l_{1}+1\right)\left(2 l_{2}+1\right)}{4 \pi}}\left(\begin{array}{ccc}
l & l_{1} & l_{2} \\
0 & 0 & 0
\end{array}\right) .
$$

Though tedious, the calculation related to $S_{1}^{\otimes}$ can be simplified using the gradient relation for spherical harmonics by raising and lowering of the spin [17]:

$$
\nabla Y_{l m}=\sqrt{\frac{l(l+1)}{2}}\left[{ }_{1} Y_{l m} \mathbf{m}_{+}-{ }_{-1} Y_{l m} \mathbf{m}_{-}\right]
$$

where

$$
\mathbf{m}_{ \pm}=\frac{1}{\sqrt{2}}\left(e_{\hat{\theta}} \mp i e_{\hat{\phi}}\right) .
$$

Combining these derivatives with the general integral in Eq. (31) leads to

$$
\begin{aligned}
I_{l m l_{1} m_{1} l_{2} m_{2}}^{\otimes}= & -\frac{i}{2} \sqrt{l_{1}\left(l_{1}+1\right) l_{2}\left(l_{2}+1\right)}(-1)^{m} \sqrt{\frac{(2 l+1)\left(2 l_{1}+1\right)\left(2 l_{2}+1\right)}{4 \pi}}\left(\begin{array}{ccc}
l & l_{1} & l_{2} \\
-m & m_{1} & m_{2}
\end{array}\right)\left(\begin{array}{ccc}
l & l_{1} & l_{2} \\
0 & -1 & 1
\end{array}\right) \\
& \times\left[1-(-1)^{\left.l+l_{2}+l_{2}\right] .}\right.
\end{aligned}
$$

Again using the orthonormality relation in Eq. (33),

$$
S_{1}^{\otimes}=\frac{1}{2 l+1}\left(F_{l l_{1} l_{2}}^{\otimes}\right)^{2}
$$

with

$$
\begin{aligned}
F_{l l_{1} l_{2}}^{\otimes}= & \frac{1}{2} \sqrt{l_{1}\left(l_{1}+1\right) l_{2}\left(l_{2}+1\right)}\left(\begin{array}{ccc}
l & l_{1} & l_{2} \\
0 & -1 & 1
\end{array}\right) \\
& \times \sqrt{\frac{(2 l+1)\left(2 l_{1}+1\right)\left(2 l_{2}+1\right)}{4 \pi}}\left[1-(-1)^{l+l_{1}+l_{2}}\right] .
\end{aligned}
$$


To calculate $S_{2}^{\otimes}$ and $S_{2}^{\oplus}$, we first note that

$$
\sum_{m_{1}} Y_{l_{1} m_{1}: a} Y_{l_{1} m_{1}: b}^{*}=\frac{l_{1}\left(l_{1}+1\right)\left(2 l_{1}+1\right)}{8 \pi} g_{a b},
$$

where $g_{a b}$ is the usual metric of unit sphere,

$$
g_{a b}=\left(\begin{array}{cc}
1 & 0 \\
0 & \sin ^{2} \theta
\end{array}\right)
$$

These allow us to show that

$$
\begin{aligned}
S_{2}^{\oplus} & \equiv \frac{1}{2} \sum_{m_{1}} J_{l m l m l_{1} m_{1} l_{1} m_{1}}^{\oplus}+\text { c.c. } \\
& =-l(l+1) l_{1}\left(l_{1}+1\right) \frac{2 l_{1}+1}{8 \pi} .
\end{aligned}
$$

Also $S_{2}^{\otimes}=S_{2}^{\oplus}$. Finally, combining all expressions, we can write

$$
\begin{aligned}
C_{l}^{\tilde{\theta}}= & C_{l}^{\theta}-l(l+1) R C_{l}^{\theta}+\sum_{l_{1} l_{2}} \frac{C_{l_{1}}^{\theta}}{2 l+1}\left[C_{l_{2}}^{h^{\oplus}}\left(F_{l l_{1} l_{2}}^{\oplus}\right)^{2}\right. \\
& \left.+C_{l_{2}}^{h^{\otimes}}\left(F_{l l_{1} l_{2}}^{\otimes}\right)^{2}\right],
\end{aligned}
$$

where $F_{l l_{1} l_{2}}^{\oplus}$ and $F_{l l_{1} l_{2}}^{\otimes}$ are given in Eqs. (35) and (40), respectively, and

$$
R=\sum_{l_{1}} l_{1}\left(l_{1}+1\right) \frac{2 l_{1}+1}{8 \pi}\left[C_{l_{1}}^{h^{\oplus}}+C_{l_{1}}^{h^{\otimes}}\right] .
$$

This expression is similar to that of Eq. (62) of Ref. [2] when $C_{l_{1}}^{h^{\diamond}}=0$ and $C_{l_{1}}^{h^{\oplus}}$ is identified as the power spectrum of projected lensing potentials due to intervening density perturbations between us and the CMB.

\section{B. Polarization}

The lensing effect on CMB polarization can be described similar to temperature anisotropies by making use of the remapping ${ }_{ \pm} \tilde{X}(\hat{\mathbf{n}})={ }_{ \pm} X(\hat{\mathbf{n}})+$ $\nabla_{i} \phi(\hat{\mathbf{n}}) \nabla^{i} X(\hat{\mathbf{n}})+\cdots+$, where ${ }_{ \pm} X=Q \pm i U$, where we have simplified the notation by replacing the spindependent gradients with a covariant derivative that acts on the spin components of the symmetric tensors that are traceless. While not explicitly stated, the rotation needed to align the polarization basis vectors between the lensed and unlensed fields is hidden in our shortened notation [18]. We refer the reader to Refs. $[18,19]$ for details of our shorthand notation and why it can be used for the lensing of the polarization pattern on the spherical sky. While our notation here is simple and follows that of Ref. [2], the final result is the same as what one gets by using the standard notation of differential geometry. This is due to the fact that the overlapping integrals involving spin harmonics that we will perform remain consistent with our simplified notation.

As is well known, the CMB polarization components form a spin-2 field and are expanded in terms of the spin- weighted spherical harmonics such that ${ }_{ \pm} X(\hat{\mathbf{n}})=$ $\sum_{l m \pm} X_{l m \pm 2} Y_{l m}(\hat{\mathbf{n}})$. Instead of Stokes parameters, the more popular $E$ and $B$ modes are given by ${ }_{ \pm} X_{l m}=E_{l m} \pm$ $i B_{l m}$. We will discuss lensing modifications to angular power spectra of $E$ - and $B$-modes as well as the cross correlation between $E$ and $\theta$, while cross correlation between $E$ and $B$, and between $B$ and $\theta$ are ignored as these are zero through parity arguments. Furthermore, the modifications to polarization by lensing do not violate parity conservation.

Taking the spherical harmonic moment of the polarization field under lensing, we write

$$
\begin{aligned}
{ }_{ \pm} \tilde{X}_{l m}= & { }_{ \pm} X_{l m}-\sum_{l_{1} m_{1} l_{2} m_{2}}\left[{ } _ { \pm } X _ { l _ { 1 } m _ { 1 } } \left({ }_{ \pm 2} I_{l m l_{1} m_{1} l_{2} m_{2}}^{\oplus} h_{l_{2} m_{2}}^{\oplus}\right.\right. \\
& \left.\left.+{ }_{ \pm 2} I_{l m l_{1} m_{1} l_{2} m_{2}}^{\otimes} h_{l_{2} m_{2}}^{\otimes}\right)\right] \\
& +\frac{1}{2} \sum_{l_{1} m_{1} l_{2} m_{2} l_{3} m_{3}}\left[{ } _ { \pm } X _ { l _ { 1 } m _ { 1 } } \left({ }_{ \pm 2} J_{l m l_{1} m_{1} l_{2} m_{2} l_{3} m_{3}}^{\oplus} h_{l_{2} m_{2}}^{\oplus} h_{l_{3} m_{3}}^{\oplus *}\right.\right. \\
& \left.\left.+{ }_{ \pm 2} J_{l m l_{1} m_{1} l_{2} m_{2} l_{3} m_{3}}^{\otimes} h_{l_{2} m_{2}}^{\otimes} h_{l_{3} m_{3}}^{\otimes *}\right)\right],
\end{aligned}
$$

where

$$
\begin{aligned}
& { }_{ \pm 2} I_{l m l_{1} m_{1} l_{2} m_{2}}^{\oplus}=\int d \hat{\mathbf{n}}\left({ }_{ \pm 2} Y_{l m}^{*}\right)\left({ }_{ \pm 2} Y_{l_{1} m_{1}}^{a}\right) Y_{l_{2} m_{2}: a} \\
& \left.{ }_{ \pm 2} J_{l m l_{1} m_{1} l_{2} m_{2} l_{3} m_{3}}^{\oplus}=\int d \hat{\mathbf{n}}\left({ }_{ \pm 2} Y_{l m}^{*}\right){ }_{ \pm 2} Y_{l_{1} m_{1}}^{a b}\right) Y_{l_{2} m_{2}: a} Y_{l_{3} m_{3}: b}^{*} \\
& { }_{ \pm 2} I_{l m l_{1} m_{1} l_{2} m_{2}}^{\otimes}=\int d \hat{\mathbf{n}}\left({ }_{ \pm 2} Y_{l m}^{*}\right)\left({ }_{ \pm 2} Y_{l_{1} m_{1}}^{a}\right) Y_{l_{2} m_{2}: b} \boldsymbol{\epsilon}_{a}^{b} \\
& { }_{ \pm 2} J_{l m l_{1} m_{1} l_{2} m_{2} l_{3} m_{3}}^{\otimes}=\int d \hat{\mathbf{n}}\left({ }_{ \pm 2} Y_{l m}^{*}\right)\left({ }_{ \pm 2} Y_{l_{1} m_{1}}^{: a b}\right) \\
& \times Y_{l_{2} m_{2}: c} Y_{l_{3} m_{3}: d}^{*} \epsilon_{a}^{c} \epsilon_{b}^{d} .
\end{aligned}
$$

After straightforward but tedious algebra, the lensed power spectra of $\tilde{E}$-modes, $\tilde{B}$-modes, and the cross correlation between $\tilde{E}$-modes and $\tilde{\theta}$ are

$$
\begin{aligned}
& C_{l}^{\tilde{E}}=C_{l}^{E}+\frac{1}{2} \sum_{l_{1} l_{2}}\left[C_{l_{1}{ }_{2}}^{h^{\oplus}} S_{1}^{\oplus}+C_{l_{1} 2}^{h_{2}} S_{1}^{\otimes}\right]\left[\left(C_{l_{2}}^{E}+C_{l_{2}}^{B}\right)\right. \\
& \left.+(-1)^{L}\left(C_{l_{2}}^{E}-C_{l_{2}}^{B}\right)\right]+C_{l}^{E} \sum_{l_{1}}\left(C_{l_{1} 2}^{h_{2}} S_{2}^{\oplus}+C_{l_{1}}^{h_{2}} S_{2}^{\otimes}\right), \\
& C_{l}^{\tilde{B}}=C_{l}^{B}+\frac{1}{2} \sum_{l_{1} l_{2}}\left[C_{l_{1} 2}^{h^{\oplus}} S_{1}^{\oplus}+C_{l_{1}}^{h^{\otimes}} S_{1}^{\otimes}\right]\left[\left(C_{l_{2}}^{E}+C_{l_{2}}^{B}\right)\right. \\
& \left.+(-1)^{L}\left(C_{l_{2}}^{B}-C_{l_{2}}^{E}\right)\right]+C_{l}^{B} \sum_{l_{1}}\left(C_{l_{1}}^{h_{2}} S_{2}^{\oplus}+C_{l_{1}}^{h_{2}} S_{2}^{\otimes}\right), \\
& C_{l}^{\tilde{\theta} \tilde{E}}=C_{l}^{\theta E}+\frac{1}{2} \sum_{l_{1} l_{2}}\left(1+(-1)^{L}\right)\left(C_{l_{1} 02}^{h^{\oplus}} S_{1}^{\oplus}+C_{l_{1}{ }_{02}}^{h^{\otimes}} S_{1}^{\otimes}\right) C_{l_{2}}^{\theta E} \\
& \left.+\frac{1}{4} C_{l}^{\theta E} \sum_{l_{1}}\left(C_{l_{1}}^{h^{\oplus}}{ }_{2} S_{2}^{\oplus}+S_{2}^{\oplus}\right)+C_{l_{1}}^{h_{2}}\left(S_{2}^{\otimes}+S_{2}^{\otimes}\right)\right),
\end{aligned}
$$

where $L=l+l_{1}+l_{2}$ and 


$$
\begin{aligned}
{ }_{2} S_{1}^{\oplus} & =\left.\left.\sum_{m_{1} m_{2}}\right|_{ \pm 2} I_{l m l_{1} m_{1} l_{2} m_{2}}\right|^{2}, \\
{ }_{2} S_{1}^{\otimes} & =\left.\left.\sum_{m_{1} m_{2}}\right|_{ \pm 2} I_{l m l_{1} m_{1} l_{2} m_{2}}^{\otimes}\right|^{2}, \\
{ }_{2} S_{2}^{\oplus} & =\frac{1}{2} \sum_{m_{1}}{ }_{ \pm 2} J_{l m l m l_{1} m_{1} l_{1} m_{1}}^{\oplus}+\text { c.c., } \\
{ }_{2} S_{2}^{\otimes} & =\frac{1}{2} \sum_{m_{1}}{ }_{ \pm 2} J_{l m l m l_{1} m_{1} l_{1} m_{1}}^{\otimes}+\text { c.c., } \\
{ }_{02} S_{1}^{\oplus} & =\sum_{m_{1} m_{2}}\left(I_{l m l_{1} m_{1} l_{2} m_{2}}^{\oplus}+{ }_{2} I_{l m l_{1} m_{1} l_{2} m_{2}}^{\oplus}\right), \\
{ }_{02} S_{1}^{\otimes} & =\sum_{m_{1} m_{2}}\left(I_{l m l_{1} m_{1} l_{2} m_{2}}^{\otimes}+{ }_{2} I_{l m l_{1} m_{1} l_{2} m_{2}}^{\otimes}\right) .
\end{aligned}
$$

To simplify terms in Eq. (49), we again make use of the integral relations outlined earlier when describing lensing of temperature anisotropies. In the case of polarization, these relations need to be generalized for integrals over spin-weighted spherical harmonics. First, the integral related to the gradient spectra is straightforward. Making use of the fact that $\nabla^{2}{ }_{ \pm 2} Y_{l m}=[-l(l+1)+4]_{ \pm 2} Y_{l m}$ and using Eq. (31), we find

$$
\begin{aligned}
{ }_{2} S_{1}^{\oplus}= & \left.\left.\frac{1}{2 l+1}\right|_{2} F_{l l_{1} l_{2}}^{\oplus}\right|^{2}, \\
{ }_{2} F_{l l_{1} l_{2}}^{\oplus}= & \frac{1}{2}\left[l_{1}\left(l_{1}+1\right)+l_{2}\left(l_{2}+1\right)-l(l+1)\right] \\
& \times \sqrt{\frac{(2 l+1)\left(2 l_{1}+1\right)\left(2 l_{2}+1\right)}{4 \pi}}\left(\begin{array}{ccc}
l & l_{1} & l_{2} \\
2 & 0 & -2
\end{array}\right) .
\end{aligned}
$$

This is exactly the relation that one encounters when lensing the polarization field by foreground density fluctuations [2].

The integral related to the curl-type displacement is tedious, but can be simplified using relations involving raising and lowering of the spin and gradient of the spinweighted spherical harmonic. To calculate

$$
{ }_{2} I_{l m l_{1} m_{1} l_{2} m_{2}}^{\otimes}=\int d \hat{\mathbf{n}}\left({ }_{ \pm 2} Y_{l m}^{*}\right)_{ \pm 2} Y_{l_{1} m_{1}: a} Y_{l_{2} m_{2}: b} \epsilon^{b a},
$$

we note

$$
\begin{aligned}
& \mathbf{m}_{-} \cdot \nabla_{s} Y_{l m}=\sqrt{\frac{(l-s)(l+s+1)}{2}}{ }_{s+1} Y_{l m} \\
& \mathbf{m}_{+} \cdot \nabla_{s} Y_{l m}=-\sqrt{\frac{(l+s)(l-s+1)}{2}}{ }_{s-1} Y_{l m},
\end{aligned}
$$

and the relation

$$
\left(\mathbf{m}_{+}\right)_{i}\left(\mathbf{m}_{-}\right)_{j}+\left(\mathbf{m}_{-}\right)_{i}\left(\mathbf{m}_{+}\right)_{j}=g_{i j}
$$

to write $\nabla_{s} Y_{l m}=\left(\mathbf{m}_{+} \mathbf{m}_{-}+\mathbf{m}_{-} \mathbf{m}_{+}\right) \cdot \nabla_{s} Y_{l m}$ as

$$
\begin{aligned}
{ }_{s} Y_{l m}= & \sqrt{\frac{(l-s)(l+s+1)}{2}}{ }_{s+1} Y_{l m} \mathbf{m}_{+} \\
& -\sqrt{\frac{(l+s)(l-s+1)}{2}}{ }_{s-1} Y_{l m} \mathbf{m}_{-} .
\end{aligned}
$$

This leads to

$$
\begin{aligned}
{ }_{2} I_{l m l_{1} m_{1} l_{2} m_{2}}^{\otimes}= & \int d \hat{\mathbf{n}}\left({ }_{+2} Y_{l m}^{*}\right)_{+2} Y_{l_{1} m_{1}: a} Y_{l_{2} m_{2}: b} \epsilon^{b a} \\
= & i(-1)^{m} \sqrt{\frac{l_{2}\left(l_{2}+1\right)}{2}} \sqrt{\frac{(2 l+1)\left(2 l_{1}+1\right)\left(2 l_{2}+1\right)}{4 \pi}}\left(\begin{array}{ccc}
l & l_{1} & l_{2} \\
-m & m_{1} & m_{2}
\end{array}\right)\left(\sqrt{\frac{\left(l_{1}+2\right)\left(l_{1}-1\right)}{2}}\left(\begin{array}{ccc}
l & l_{1} & l_{2} \\
2 & -1 & -1
\end{array}\right)\right. \\
& \left.-\sqrt{\frac{\left(l_{1}-2\right)\left(l_{1}+3\right)}{2}}\left(\begin{array}{ccc}
l & l_{1} & l_{2} \\
2 & -3 & 1
\end{array}\right)\right),
\end{aligned}
$$

such that

$$
\begin{aligned}
{ }_{2} S_{1}^{\otimes} & =\left.\left.\sum_{m_{1} m_{2}}\right|_{2} I_{l m l_{1} m_{1} l_{2} m_{2}}^{\otimes}\right|^{2}=\left.\left.\frac{1}{2 l+1}\right|_{2} F_{l_{1} l_{2}}^{\otimes}\right|^{2} \\
{ }_{2} F_{l l_{1} l_{2}}^{\otimes} & =\sqrt{\frac{l_{2}\left(l_{2}+1\right)(2 l+1)\left(2 l_{1}+1\right)\left(2 l_{2}+1\right)}{8 \pi}}\left(\sqrt{\frac{\left(l_{1}+2\right)\left(l_{1}-1\right)}{2}}\left(\begin{array}{ccc}
l & l_{1} & l_{2} \\
2 & -1 & -1
\end{array}\right)-\sqrt{\frac{\left(l_{1}-2\right)\left(l_{1}+3\right)}{2}}\left(\begin{array}{ccc}
l & l_{1} & l_{2} \\
2 & -3 & 1
\end{array}\right)\right) .
\end{aligned}
$$


Furthermore, with $s= \pm 2$, Eq. (43) can be generalized to

$$
{ }_{2} S_{2}^{\oplus}=-\frac{1}{2}[l(l+1)-4] l_{1}\left(l_{1}+1\right) \frac{2 l_{1}+1}{4 \pi},
$$

and as in the case of lensed temperature anisotropies,

$$
{ }_{+2} S_{2}^{\otimes}={ }_{+2} S_{2}^{\oplus} .
$$

For the cross correlation between $E$-modes and the tem- perature, we find

$$
\begin{aligned}
& { }_{02} S_{1}^{\oplus}=\frac{1}{2 l+1}\left(F_{l l_{1} l_{2}}^{\oplus}\right)\left({ }_{+2} F_{l l_{1} l_{2}}^{\oplus}\right) \\
& { }_{02} S_{1}^{\otimes}=\frac{1}{2 l+1}\left(F_{l l_{1} l_{2}}^{\otimes}\right)\left({ }_{+2} F_{l l_{1} l_{2}}^{\otimes}\right) .
\end{aligned}
$$

Putting the terms together, we can write the lensed power spectra in polarization as

$$
\begin{aligned}
C_{l}^{\tilde{E}} & \left.=C_{l}^{E}-\left(l^{2}+l-4\right) R C_{l}^{E}+\frac{1}{2(2 l+1)} \sum_{l_{1} l_{2}}\left[C_{l_{1}}^{h^{\oplus}}{ }_{2} F_{l l_{1} l_{2}}\right)^{2}+C_{l_{1}}^{h^{\otimes}}\left({ }_{2} F_{l l_{1} l_{2}}^{\otimes}\right)^{2}\right]\left[\left(C_{l_{2}}^{E}+C_{l_{2}}^{B}\right)+(-1)^{L}\left(C_{l_{2}}^{E}-C_{l_{2}}^{B}\right)\right] \\
C_{l}^{\tilde{B}} & =C_{l}^{B}-\left(l^{2}+l-4\right) R C_{l}^{B}+\frac{1}{2(2 l+1)} \sum_{l_{1} l_{2}}\left[C_{l_{1}}^{h^{\oplus}}\left({ }_{2} F_{l l_{1} l_{2}}\right)^{2}+C_{l_{1}}^{h_{2}}\left({ }_{2} F_{l l_{1} l_{2}}^{\otimes}\right)^{2}\right]\left[\left(C_{l_{2}}^{E}+C_{l_{2}}^{B}\right)-(-1)^{L}\left(C_{l_{2}}^{E}-C_{l_{2}}^{B}\right)\right] \\
C_{l}^{\tilde{\theta} \tilde{E}} & =C_{l}^{\theta E}-\left(l^{2}+l-2\right) R C_{l}^{\theta E}+\frac{1}{2 l+1} \sum_{l_{1} l_{2}}\left[C_{l_{1}}^{h^{\oplus}}\left(F_{l l_{1} l_{2}}^{\oplus}\right)\left({ }_{+2} F_{l l_{1} l_{2}}^{\oplus}\right)+C_{l_{1}}^{h^{\otimes}}\left(F_{l l_{1} l_{2}}^{\otimes}\right)\left({ }_{+2} F_{l l_{1} l_{2}}\right)\right] C_{l_{2}}^{\theta E},
\end{aligned}
$$

with $R$ from Eq. (45).

The case of CMB lensing by foreground density fluctuations is simply the replacement of $C_{l_{1}}^{h^{\oplus}}$ with the power spectrum of projected potentials and with $C_{l_{1}}^{h^{\diamond}}=0$. The lensing contribution to the $B$-mode from the foreground density field [5] acts as the main contaminant in detecting the primary gravitational wave signal in $B$-modes of polarization. To see the extent to which lensing by gravitational waves themselves may become important in $B$-mode polarization studies, we will assume $C_{l}^{B}$ to be zero and present a comparison between $C_{l}^{\tilde{B}}$ from density fluctuations and $C_{l}^{\tilde{B}}$ from gravitational waves. As we find, the secondary lensing from gravitational waves is smaller than the cosmic variance level of lensing $B$-modes from density perturbations and will remain undetectable in anisotropy maps. While below the cosmic variance limit, $C_{l} / \sqrt{l}$, for anisotropy measurements, systematics must generally be controlled to a level far below this; if systematics apply to a wide range of multipoles, then one must control their effects to $C_{l} / l$. For lensing by foreground gravitational waves at the maximum amplitude, we find that the corrections are above this level when $l>10^{3}$ suggesting that one only needs to be concerned of these signals in all-sky maps with no instrumental noise and other secondary signals.

\section{RESULTS AND DISCUSSION}

In Fig. 1, we show a comparison of $C_{l}^{h^{\otimes}}, C_{l}^{h^{\oplus}}$, and the angular power spectrum of deflection angle from projected density perturbations along the line of sight to $\eta_{s}$ at a redshift of 1100 corresponding to the CMB last scattering surface. In calculating the power spectra of lensing from foreground gravitational waves, we have assumed an amplitude for the tensor modes with a value for $H_{I}$ in Eq. (15) of $2 \times 10^{14} \mathrm{GeV}$. This corresponds to a tensor-to-scalar ratio of 0.3 , which is roughly the upper limit allowed by current CMB and large-scale structure observations [20].

The curl spectrum of deflections from gravitational waves has been previously discussed in the literature in the context of weak lensing surveys with galaxy shapes [10]. The gradient-type displacement spectrum from gravitational waves discussed here is also important and cannot be ignored when calculating modifications to CMB temperature and polarization anisotropies. Note that $C_{l}^{h^{\diamond}}$ and $C_{l}^{h^{\oplus}}$ peak at large angular scales corresponding to $\ell=2$ to $\ell=10$. To compare lensing by gravitational waves and lensing by mass, we calculate the rms deflection angle

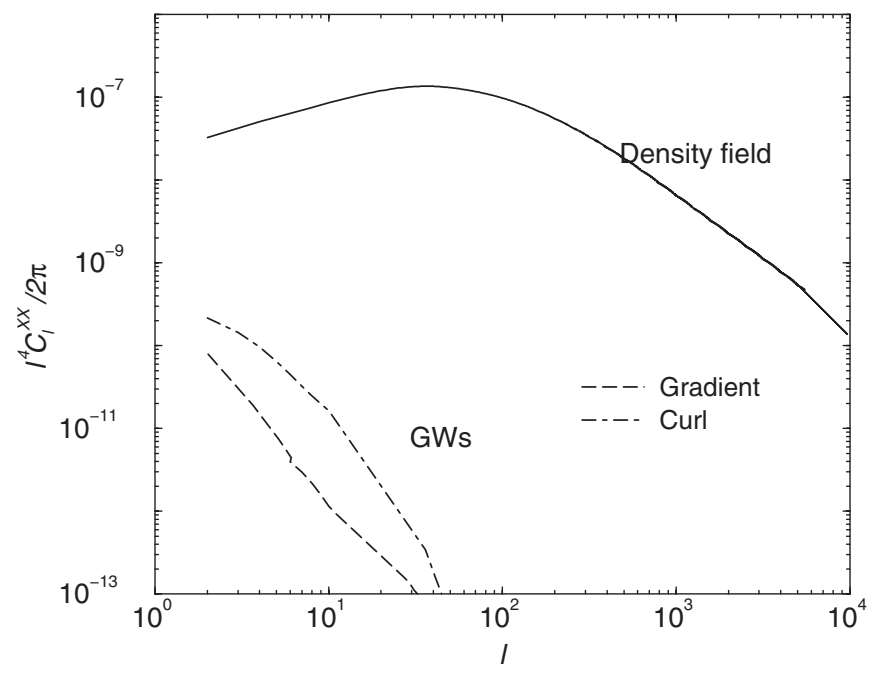

FIG. 1. Lensing-deflection power spectra. Here, we show the gradient component from density perturbations (top curve), the curl (dot-dashed line) and gradient (dashed line) components from foreground inflationary gravitational waves. We have taken a background of gravitational waves with an amplitude for the power spectrum corresponding to roughly a tensor-to-scalar ratio of 0.3 or a Hubble parameter during inflation of $2 \times 10^{14} \mathrm{GeV}$. 
through $\theta_{\mathrm{rms}}^{2}=\left[\sum l(l+1)(2 l+1) / 4 \pi\left(C_{l}^{h^{\otimes}}+C_{l}^{h^{\oplus}}\right)\right]$. For gravitational waves related spectra shown in Fig. $1, \theta_{\text {rms }}$ is $3.62 \times 10^{-5}$ radians or roughly 7 arcsecs, which is a factor of 20 smaller than the rms deflection angle for CMB photons under density fluctuations, where $\theta_{\text {rms }} \sim 7 \times$ $10^{-4}$. The coherence scale, where the rms drops to half of its peak value, is about $\sim 60^{\circ}$ for lensing by gravitational waves, while for density perturbations the coherence scale is about a degree. With such a large coherence scale and a small rms deflection angle, foreground gravitational waves deflect large patches of the CMB sky by the same angle of about 6 arcsecs, resulting in an overall small modification to the CMB anisotropy and polarization spectra, when compared to the case with density fluctuations alone.

The difference between lensing by density perturbations and lensing by gravitational waves is clear in Fig. 2, where we show modifications to the temperature power spectrum and the cross power spectrum between temperature and $E$-modes of polarization. The secondary lensing correction from the foreground gravitational waves is smaller than the cosmic variance level of intrinsic CMB anisotropies. Even with perfect $\mathrm{CMB}$ observations devoid of instrumental noise, it is unlikely that the lensing modification by primordial gravitational waves in the foreground of $\mathrm{CMB}$ will be detectable. As shown in Fig. 2, however, the corrections are above the systematic level of $C_{l} / l$ for primordial anisotropy measurements when $l>10^{3}$. Thus, in the extreme case where one is dealing with perfect all-sky maps

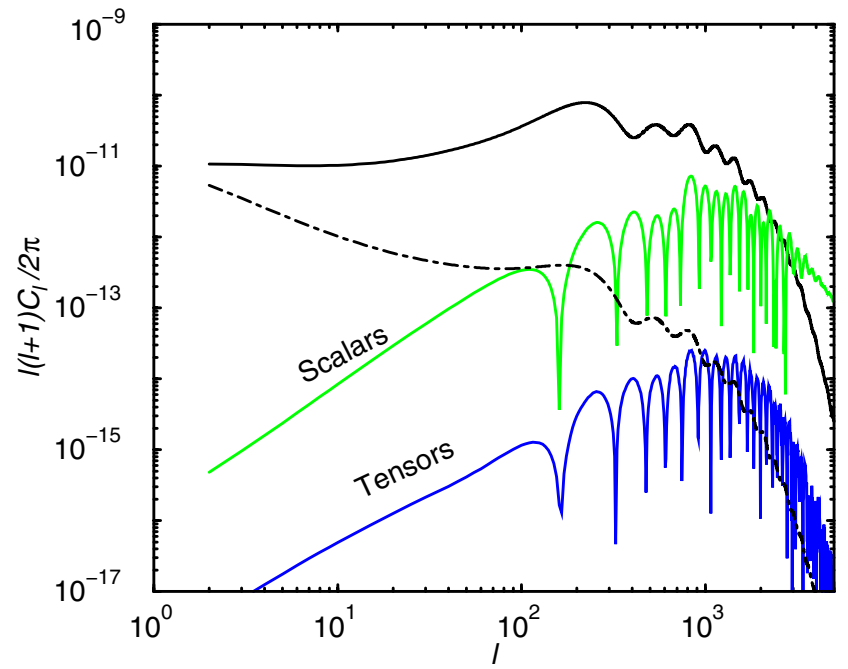

cleaned of foregrounds and other secondary signals, one could be concerned that these effects act as a source of systematic error for primordial anisotropy measurements. As is clear from Fig. 2, while lensing by gravitational waves is not significant, lensing of the CMB by foreground density perturbations will be detectable as the modifications are well above the cosmic variance limit.

In Fig. 3, we summarize our results related to lensing of polarization anisotropies in terms of the power spectra or $E$ - and $B$-modes. Again, the lensing effect by foreground gravitational waves is below the cosmic variance level of the dominant signal in the $E$ - and $B$-mode maps, but above the systematic level. In the case of $B$-modes, lensing by density perturbations will remain the main contaminant in searching for gravitational wave signatures from the primary $B$-mode power spectrum. Lensing signals by foreground gravitational waves are unlikely to affect reconstruction techniques that attempt to remove the lensed $B$-modes when searching for a low amplitude gravitational wave background [4].

While we have only considered angular displacements on the sky, gravitational waves also contribute to a variation along the line of sight that can be described as a timedelay effect. Just as angular displacement couples to the angular gradient of the $\mathrm{CMB}$, the radial displacement couples to the radial gradient of the CMB. These effects, however, are smaller due to lack of radial structure in the perturbations that form the primordial anisotropy spectrum

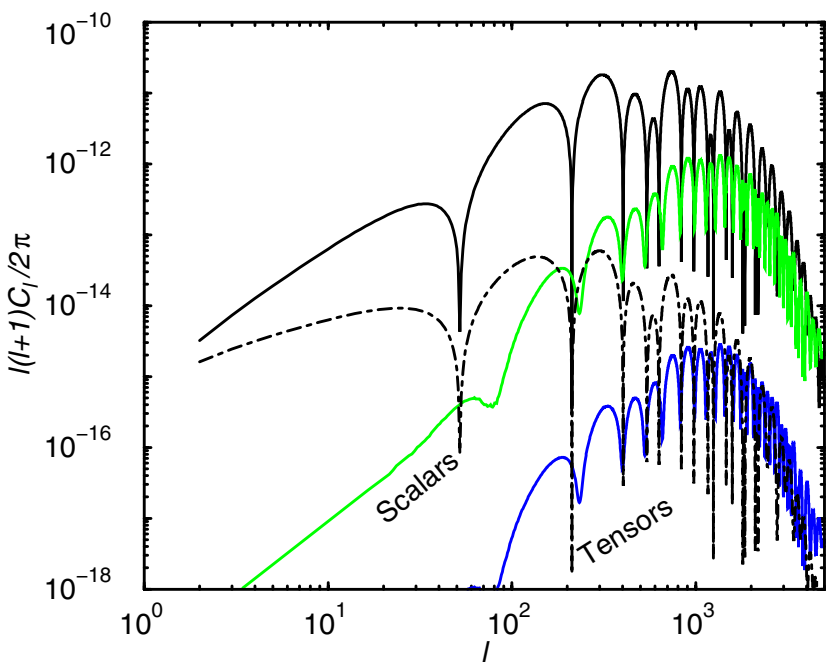

FIG. 2 (color online). The lensing modification to CMB power spectra for density perturbations and for gravitational waves. Left: Temperature fluctuations. The top curve is the primordial power spectrum. The middle curve is the secondary anisotropy contribution, $\left|\tilde{C}_{l}-C_{l}\right|$, to the temperature power spectrum from lensing by density perturbations, and the lower curve is for lensing by gravitational waves, assuming the maximum inflationary gravitational waves background is consistent with current data with the deflection power spectra shown in Fig. 1. Right: Temperature-E polarization cross correlation, with curves following the left panel. In both panels, the thin long-dashed line is $C_{l} / l$ at each multipole with $C_{l}$ related to the intrinsic anisotropy spectrum; while the cosmic variance is $C_{l} / \sqrt{l}$, $C_{l} / l$ denotes the level at which one must control the systematics, if the effects resulting systematics apply to a wide range of multipoles. The lensing by density fluctuations cannot be ignored as the corrections are well above the cosmic variance limit and will be detectable in upcoming anisotropy data. The lensing by foreground gravitational waves, however, are below the cosmic variance limit, suggesting that they will remain undetectable, but above the systematic level when $l>10^{3}$. 


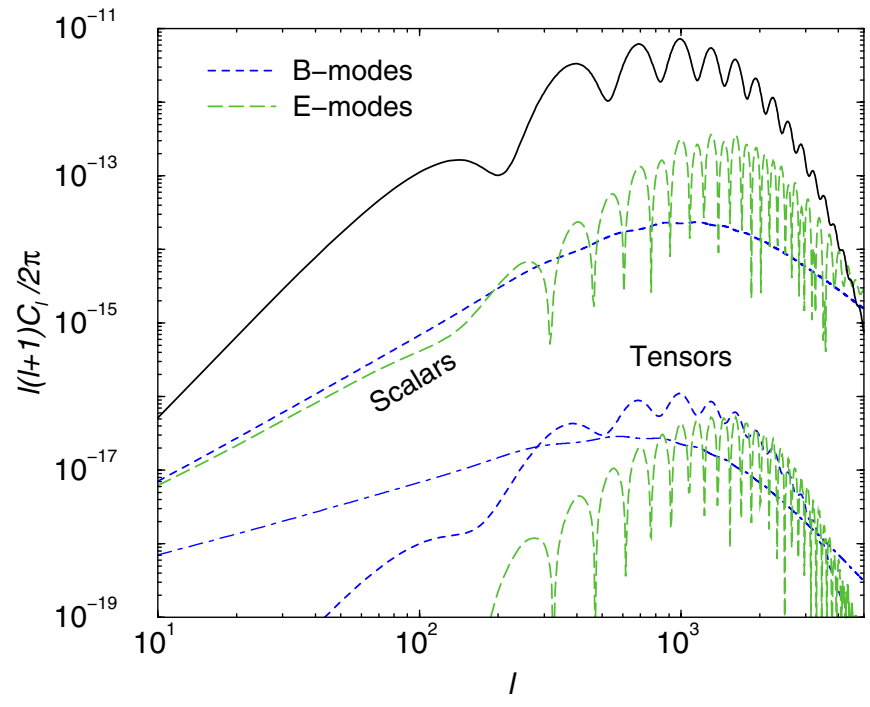

FIG. 3 (color online). The lensing modification to CMB polarization spectra for density perturbations and for gravitational waves. From top to bottom, these curves are for primordial power spectrum in the $E$-mode (solid line), lensing correction to $E$-mode from density fluctuations (long-dashed line), lensing correction to $B$-mode from density fluctuations (short-dashed line labeled "Scalars"), lensing correction to $B$-mode from gravitational waves (short-dashed line labeled "Tensors"), and the lensing correction to $E$-mode from gravitational waves (longdashed line). For reference, the thin dot-dashed line is the systematic level of the $B$-mode lensing power spectrum, $C_{l} / l$, from the density field. While the corrections from lensing by foreground gravitational waves is below the cosmic variance limit of the lensing $B$-mode power spectrum and, again, undetectable in anisotropy maps, they may become a source of systematic in all-sky maps with no instrumental noise and other secondary signals and foregrounds.

in the $\mathrm{CMB}$, such as the acoustic peaks. There are also geometric cancellations associated with the projection of line-of-sight time-delay modulations to an anisotropy pattern on the CMB sky [15]. Thus, it is unlikely that our conclusions related to lensing by foreground gravitational waves are affected by including the time-delay effect.

In general, our results are consistent with those of Ref. [10] who studied the possibility of measuring the gravitational wave background amplitude using weak lensing surveys of galaxy shapes and using the curl mode of the shear. Even with an optimistic survey with a large surface density of galaxies to measure shapes, the gravitational wave signal in the shear remains undetectable below the noise.

To summarize our calculation, while weak lensing distortion of the cosmic microwave background (CMB) temperature and polarization patterns by foreground density fluctuations is well studied in the literature, we noted the lack of a detailed description related to lensing modifications by foreground gravitational waves or tensor perturbations. Here, we have presented an analytical formulation on how CMB anisotropies and polarization patterns are distorted by a stochastic background of primordial gravitational waves between us and the last scattering surface. Our analytical formulation is useful when studying general lensing of any background source by foreground gravitational waves.

While density fluctuations perturb CMB photons via gradient-type displacements only, gravitational waves distort CMB anisotropies via both the gradient- and the curltype displacements. The latter can be described as a rotation of background images in the presence of foreground gravitational waves while the former is related to the lensing convergence. For a primordial background of gravitational waves from inflation with an amplitude corresponding to a tensor-to-scalar ratio below the current upper limit of $\sim 0.3$, the resulting modifications to the angular power spectra of $\mathrm{CMB}$ temperature anisotropy and polarization are below the cosmic variance limit, but above the systematic level. Thus, it is unlikely that planned high sensitivity CMB observations warrant an accounting of the secondary contributions discussed here as they are not expected to affect precise parameter measurements; if observations are all-sky measurements with no instrumental noise, then these effects may be present in the form of systematic corrections to the primary anisotropy and polarization measurements.

\section{ACKNOWLEDGMENTS}

We thank A. Stebbins and J. Pritchard for useful discussions. This work was supported in part by DOE at UC Irvine (A.C.) and by the Moore Foundation at Caltech (C. L.).
[1] See, e.g., U. Seljak and M. Zaldarriaga, Phys. Rev. Lett. 82, 2636 (1999); Phys. Rev. D 60, 043504 (1999); M. Zaldarriaga and U. Seljak, Phys. Rev. D 59, 123507 (1999); W. Hu, Phys. Rev. D 64, 083005 (2001).

[2] W. Hu, Phys. Rev. D 62, 043007 (2000).

[3] A. Lewis and A. Challinor, Phys. Rep. 429, 1 (2006).
[4] W. Hu and T. Okamoto, Astrophys. J. 574, 566 (2002); M. Kesden, A. Cooray, and M. Kamionkowski, Phys. Rev. D 67, 123507 (2003); C. M. Hirata and U. Seljak, Phys. Rev. D 68, 083002 (2003).

[5] M. Zaldarriaga and U. Seljak, Phys. Rev. D 58, 023003 (1998). 
[6] M. Kamionkowski, A. Kosowsky, and A. Stebbins, Phys. Rev. Lett. 78, 2058 (1997); U. Seljak and M. Zaldarriaga, Phys. Rev. Lett. 78, 2054 (1997).

[7] M. Kesden, A. Cooray, and M. Kamionkowski, Phys. Rev. Lett. 89, 011304 (2002); L. Knox and Y.-S. Song, Phys. Rev. Lett. 89, 011303 (2002); U. Seljak and C. Hirata, Phys. Rev. D 69, 043005 (2004).

[8] N. Kaiser and A. H. Jaffe, Astrophys. J. 484, 545 (1997).

[9] A. Stebbins, astro-ph/9609149.

[10] S. Dodelson, E. Rozo, and A. Stebbins, Phys. Rev. Lett. 91, 021301 (2003).

[11] A. Cooray, M. Kamionkowski, and R. R. Caldwell, Phys. Rev. D 71, 123527 (2005).

[12] A. Cooray and W. Hu, Astrophys. J. 574, 19 (2002); C. Shapiro and A. Cooray, J. Cosmol. Astropart. Phys. 03 (2006) 007; C. M. Hirata and U. Seljak, Phys. Rev. D 68, 083002 (2003).
[13] J. Miralda-Escude, Astrophys. J. 380, 1 (1991); R. D. Blandford, A. B. Saust, T. G. Brainerd, and J. Villumsen, Astrophys. J. 251, 600 (1991); N. Kaiser, Astrophys. J. 388, 272 (1992). For recent reviews, see M. Bartelmann and P. Schneider, Phys. Rep. 340, 291 (2001); P. Schneider, Gravitational Lensing: Strong, Weak \& Micro, Lecture Notes of the 33rd Saas-Fee Advanced Course (Springer-Verlag, Berlin, 2006).

[14] I. I. Shapiro, Phys. Rev. Lett. 13, 789 (1964).

[15] W. Hu and A. Cooray, Phys. Rev. D 63, 023504 (2001).

[16] J. R. Pritchard and M. Kamionkowski, Ann. Phys. (N.Y.) 318, 2 (2005).

[17] J. N. Goldberg et al., J. Math. Phys. (N.Y.) 8, 2155 (1967).

[18] A. Challinor and G. Chon, Phys. Rev. D 66, 127301 (2002).

[19] T. Okamoto and W. Hu, Phys. Rev. D 67, 083002 (2003).

[20] D. N. Spergel et al., astro-ph/0603449. 